\title{
Array Pattern Synthesis Using 4-bit Discrete Digital Shifters by Classic Particle Swarm Optimization
}

\author{
Dong Liu ${ }^{1,2} \mathrm{a}^{*}$, Wan Mu Han ${ }^{1}$ and Qi Long Jiang ${ }^{1}$ \\ ${ }^{1}$ School of Electrical Engineering, Southwest Jiaotong University, Chengdu, Sichuan 610031, China \\ ${ }^{2}$ Electrical and Computer Engineering Department, Texas A\&M University, \\ College Station, TX, 77845, U.S. \\ aliudong@swjtu.edu.cn
}

Keywords: Antenna array; Digital phase control; Particle swarm optimization; Patterns synthesis

\begin{abstract}
In this paper, the classic PSO algorithm is applied to the pattern synthesis of antenna array using 4-bit digital phase shifters. In order to directly apply the existed PSO algorithms to the pattern synthesis using digital phase shifters, this paper proposes a simple truncation rounded number strategy to transfer the discrete problem to the continuous one, which not only produces no quantization error, but also avoids the limitation of the discrete PSO algorithm. The optimized results are better than that of the discrete PSO algorithm.
\end{abstract}

\section{Introduction}

Synthesis of linear antenna arrays, of main target is to find appropriate excitation vector and layout of the elements to generate desirable radiation pattern, is a classic optimization problem in electromagnetic $^{[1]}$.

Only phase shift of every array element is needed to conduct the synthesis of antenna array patterns by phase control. Different array elements shares the equal amplitude excitation and the intervals between each array element keep invariant, which can simplify the antenna array feed network, cut the cost and reduce the array element coupling produced by the large amplitude excitation[1]. Currently, antenna array patterns based on phase control become a heated research aspect. Many works try to realize the synthesis of antenna array patterns by phase, such as beam forming, interference suppression and so on [1-10]. Due to the technical and cost, discrete digital phase shifter is used more widely than continuous one [2-4].

PSO algorithm is an evolution algorithm based on the swarm [11], which has been broadly used in the synthesis of antenna array patterns [3,5,12-14]. For a phase control antenna array using discrete digital phase shifter, classic PSO algorithm which aims at continuous optimization problem cannot be directly applied. In order to solve this problem, in [3] , Binary PSO and Quantum PSO are applied to the pattern synthesis of 100-element and 200-element phase control arrays and in [4] Quantum IFT (QIFT) is applied to the pattern synthesis of phase control arrays with different element quantity and with different bits phase shifter.

Continuous method can also be adopted to solve the antenna array pattern synthesis using discrete digital phase shifters, but it will produce the quantization error. [2,4] investigates the quantization error of the results of antenna array pattern synthesis using discrete digital phase shifters and proposes the method on reducing it. While by using discrete PSO algorithm, the quantization error produced by the rounded results of continuous algorithm can be avoided. However, the optional algorithms will be limited and the existing PSO variations and their application cannot be applied.

In this paper, a novel algorithm combining rounded number strategy with classic PSO method is proposed. Also the impact on the probability of the discrete point values using different rounded number strategies and the basis of selecting rounded number strategy are analyzed. Finally, the proposed strategy is applied to the synthesis of antenna array patterns using 4-bit discrete digital shifters with 100 and 200 array elements respectively. Optimized results shows via our transformation, 
classic PSO can directly applied to the antenna array pattern synthesis using discrete digital phase shifters. Comparisons on optimized results and optimization efficiency between our work and existing results are also made.

\section{Digital Phase Antenna Array}

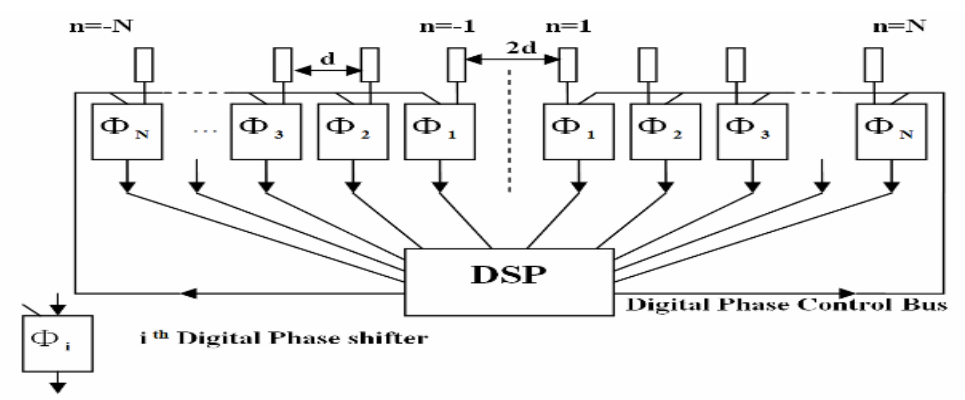

Figure. 1 Centro-symmetric antenna array using digital phase

Consider a linear phase control array antenna consisting of $2 \mathrm{~N}$ independent array elements with equal amplitude excitation, and the array elements are symmetric about the origin. Also from the origin, the intervals between each array element are all equal to d, as Fig.1 shows in [3].

For the linear array with the geometry construction as Fig. 1, its pattern expression follows:

$$
A F(\theta, I, x, \phi)=\sum_{i=-N}^{-1} I_{i} \mathrm{e}^{\mathrm{j}\left(\frac{2 \pi}{\lambda} \mathrm{x}_{\mathrm{i}} \sin (\theta)+\phi_{i}\right)}+\sum_{i=1}^{N} I_{i} \mathrm{e}^{\mathrm{j}\left(\frac{2 \pi}{\lambda} \mathrm{x}_{\mathrm{i}} \sin (\theta)+\phi_{i}\right)}
$$

where Ii and $\varphi \mathrm{i}$ are the excitation amplitude and phase of the element located at $x i$. $\lambda$ is the wavelength.

Same as [3], when classic PSO is applied to the pattern synthesis of phase control array, it must be guaranteed that the particle positions and these 16 discrete values are strict correspondence. Classic PSO algorithm only aims at the continuous optimization problems and cannot be directly extended to the discrete problems. For this reason, in [13,15], Quantum PSO and Binary PSO are proposed aiming at the discrete problems, and in [3] these two algorithms are applied to the antenna array pattern synthesis using discrete digital phase shifters.

\section{Optimization strategy and Fitness Function}

Binary PSO is more appropriate for combinatorial problem, such as TSP problem. Quantum PSO is suit for discrete value optimization problem, especially when no mapping can be established between continuous space and discrete space. In this paper, although the values of phase control array using digital phase shifters are discrete, yet a simple rounded strategy can establish a mapping between continuous space and discrete space, so that the discrete optimization problem can be transferred to a continuous one.

Truncation rounded method is to cut off the continuous value and round it to its rounded integer, by removing its decimal part and mapping range $[\mathrm{i}, \mathrm{i}+1)$ to a discrete value $\mathrm{i}$. While $(\mathrm{i}-0.5, \mathrm{i}+0.5)$ is mapped to i in classic round method.

PSO algorithm is a random optimization algorithm, with the following velocity updating equation:

$$
V_{i j}(t+1)=W V_{i j}(t)+c_{1} r_{1 j}(t)\left(p_{i j}-x_{i j}(t)\right)+c_{2} r_{2 j}(t)\left(p_{g j}-x_{i j}(t)\right)(2)
$$

The velocity updating equation is the kernel part of PSO and the unsuitable modification will make the performance worse. In this paper, the round strategy is only used in a new position value before computing the fitness value, and there is no modification in PSO operation, so the performance of PSO is no change and it still can handle with discrete optimization problems.

When applying PSO to the pattern synthesis of phase control array, it also needs an appropriate fitness function, which is used to evaluate the performance of every set of phase vector so as to guide the swarm to search in the direction of satisfying the optimization index. In this paper, the pattern 
synthesis is mainly applied to the suppression of sidelobe level and null steering. Equation (3) will be adopted when only the suppression of sidelobe level is proceeding.

$$
f(x)=\operatorname{PSLL}(A F(\theta, \phi))
$$

\section{Simulation Result}

Both in [3] and [4], 4-bit digital phase shifter was applied to optimize the antenna array patterns. To verify the proposed algorithm in this paper, only the data of the same angle resolution ration in [3] is compared. In the following simulations, the range of phase values is set to $\left[0 \sim 114.5^{\circ}\right]$ and the angle step is also set to $7.63^{\circ}$.

Consider the phase control arrays of 200 independent elements, with orientation shown as Fig. 1, half wavelength interval, 4-bit phase shifters and equal extinction amplitude. The LDWPSO proposed in [16] is applied to the optimization, with $\omega \in[0.9,0.4], \mathrm{c} 1=\mathrm{c} 2=1.5,100$ particles and 50 simulation iterations. The search range of the PSO particle is $[0,16)$. The coordinate value of the particle position in every dimension is cut off and rounded to the shifter value which will be multiplied by the stepping $7.63^{\circ}$ to get every array element phase value.

In this example, the objective of the pattern synthesis is to realize the suppression of sidelobe level in 200-element phase control array, only using the 4-bit phase shifters. Equation (3) can be set as the fitness function and the resolution ratio is set to $0.2^{\circ}$.

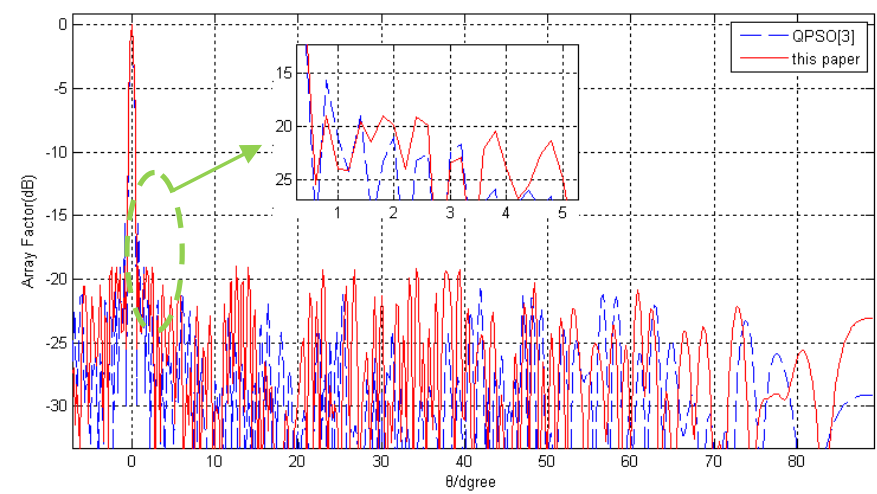

Figure. 2 The optimized array pattern with minimum PSLL only (solid)

200 elements. PSI $\mathrm{I}=-18.65 \mathrm{~dB}$

The result of the 200-element array pattern synthesis is shown in Fig. 2, where the full line is the optimal of our optimized result and the dashed line is the optimal using QPSO in [3]. The optimized result in this paper appears $-22 \mathrm{~dB}$, better than $-21 \mathrm{~dB}$ optimized in [3] in the same condition. When the angle resolution ratio is set to $0.3^{\circ}$, the optimized result in this paper increases to $-22.5 \mathrm{~dB}$, which is superior to the resultoptimized in [3], i. e. In Fig.2, the main beam width of optimized resutl in this paper is $1.2^{\circ}$.

\section{Conclusion}

For the antenna array using discrete digital phase shifters, classic PSO algorithms cannot be applied directly. Because the value point of discrete digital phase shifters is the adjacent integer, a simple rounded strategy is proposed to transfer the discrete optimization problem to a continuous one, needless of using Binary PSO and Quantum PSO. By the proposed strategy, the existing PSO research results can directly applied to the antenna array pattern synthesis using discrete digital phase shifters, which will not only produces no quantization error, but also meanwhile avoids the limitation of the discrete PSO algorithm.

In this paper, the pattern synthesis with sidelobe level suppression of 4-bit phase controled arrays with 200 elements is simulated. LDWPSO is applied to optimize the pattern and the optimized results is better than that using the Quantum PSO algorithm. 


\section{Reference}

[1] C. Lin, et al., "Synthesis of unequally spaced antenna arrays by using differential evolution," IEEE Transactions on Antennas and Propagation, vol. 58, pp. 2553-2561, 2010.

[2] M. Smith and Y. Guo, "A comparison of methods for randomizing phase quantization errors in phased arrays," Antennas and Propagation, IEEE Transactions on, vol. 31, pp. 821-828, 1983.

[3] T. H. Ismail and Z. M. Hamici, "Array Pattern Synthesis Using Digital Phase Control by Quantized Particle Swarm Optimization," Antennas and Propagation, IEEE Transactions on, vol. 58, pp. 2142-2145, 2010.

[4] W. Keizer, "Low Sidelobe Phased Array Pattern Synthesis with Compensation for Errors due to Quantized Tapering," Antennas and Propagation, IEEE Transactions on, IEEE early access.

[5] D. W. Boeringer and D. H. Werner, "Particle swarm optimization versus genetic algorithms for phased array synthesis," IEEE Transactions on Antennas and Propagation, vol. 52, pp. 771-779, 2004.

[6] J. A. Hejres, "Null steering in phased arrays by controlling the positions of selected elements," IEEE Transactions on Antennas and Propagation,, vol. 52, pp. 2891-2895, 2004.

[7] J. Bregains, et al., "Perturbation of the phases of Taylor field samples in the synthesis of linear and circular array antennas," IEEE Antennas and Wireless Propagation Letters, , vol. 3, pp. 38-40, 2004.

[8] R. Vescovo, "Reconfigurability and beam scanning with phase-only control for antenna arrays," IEEE Transactions on Antennas and Propagation, vol. 56, pp. 1555-1565, 2008.

[9] P. Rocca, et al., "Sidelobe reduction through element phase control in uniform subarrayed array antennas," IEEE Antennas and Wireless Propagation Letters, vol. 8, pp. 437-440, 2009.

[10]M. P. Daly and J. T. Bernhard, "Directional modulation technique for phased arrays," IEEE Transactions on Antennas and Propagation,vol. 57, pp. 2633-2640, 2009.

[11]J. Kennedy and R. Eberhart, "Particle swarm optimization," in Proceedings of the 1995 IEEE International Conference on Neural Networks. Part 1 (of 6), Perth, Aust, 1995, pp. 1942-1948.

[12]S. K. Goudos, et al., "Application of a comprehensive learning particle swarm optimizer to unequally spaced linear array synthesis with sidelobe level suppression and null control," IEEE Antennas and Wireless Propagation Letters, , vol. 9, pp. 125-129, 2010.

[13]N. Jin and Y. Rahmat-Samii, "Advances in particle swarm optimization for antenna designs: Real-number, binary, single-objective and multiobjective implementations," IEEE Transactions on Antennas and Propagation,, vol. 55, pp. 556-567, 2007.

[14]D. Liu, et al., "Synthesis of unequally spaced antenna arrays by using inheritance learning particle swarm optimization," Progress In Electromagnetics Research - PIER, vol. 118, pp. 205-221, 2011.

[15] J. Kennedy and R. C. Eberhart, "A discrete binary version of the particleswarm algorithm," in IEEE Int. Conf. Man Syst., Cyber., Oct.1997, vol. 5, pp. 4104-4108.

[16] Shi, Y. and R. Eberhart , "A modified particle swarm optimizer." Applied Mathematics and Computation, vol. 189, no. 2, pp: 69-73, 1998. 